\title{
IERY HIGH ENERGY $\gamma$-RAY GENERATION NEAR THE LIGHT CYLINDER OF AN AXISYMMETRIC ROTATOR: COS-B LIKE $\gamma$-RAY SOURCES
}

\author{
S. V. Bogovalov and Yu. D. Kotov \\ Moscow Engineering Physics Institute
}

\begin{abstract}
Super-hard $\gamma$-ray radiation spectra have been calculated. This radiation is generated near the velocity-of-light cylinder through the process of inverse-Compton scattering of relativistic electrons by thermal photons radiated by a neutron star. These calculations have been compared with observations of the Crab and Vela pulsars at $1000-\mathrm{GeV} \gamma$-ray energies. A correlation between $\gamma$-ray flares and those in soft $\left(E_{\mathrm{X}} \simeq 1 \mathrm{keV}\right) \mathrm{X}$-rays are predicted.
\end{abstract}

\section{ntroduction}

;atistically meaningful photon fluxes with $E_{\gamma}>1$ $2 \mathrm{~V}$ have been observed from only a few sources Veekes 1988). Two radio pulsars are among them. he generation of such high energy photons in rao pulsars with surface magnetic fields $\simeq 10^{12} \mathrm{G}$ ust occur at a considerable distance from the neuon star. Only then will the processes which con:rt $\gamma$ quanta to $e^{+} e^{-}$pairs in the magnetic field st prevent the high energy radiation from leav$g$ the source. For the faster rotating pulsars (i.e., le Crab and V'ela) the region of super-hard $\gamma$-ray ansparency begins in the vicinity of the light cylin2r. The radius of the light cylinder is $\Omega / c$, where is the frequency of pulsar rotation.

Therefore, effective acceleration must occur ose to the light cylinder. All currently existing odels in which particle acceleration takes place in te inner magnetosphere at distances small with reect to the light cylinder (Ruderman and Suthernd 1975, Arons 1983c, Cheng, Ho, and Ruderman 386b) fail to account for the flux of energetic parcles observed in the Crab and Vela pulsars. For lese pulsars the total power carried away by relavistic particles may be comparable with the rate of itational energy loss as the pulsar gradually spins own.

\section{'article acceleration}

heoretical analysis indicates that the electromagetic field is the source of energy for particle acsleration near the light cylinder. An important rcumstance was noted by Beskin, Gurevich and tomin (1983): For pulsars like the Crab and Vela, le energy carried by the azimuthal magnetic field enerated by pulsar rotation exceeds by several orers of magnitude the total kinetic energy of the $+e^{-}$plasma produced in the polar-cap region. The energy losses caused by ejection of this azimuthal magnetic field from the magnetosphere are

$$
L=H^{2} R^{6} \Omega^{4} / 4 c^{3},
$$

where $H$ is the magnetic field at the pulsar surface and $R$ is the pulsar radius. Eq.(1) assumes that the rotation axis and magnetic moment are parallel and even for this case the acceleration of particles is effective near the light cylinder (Bogovalov 1989, Bogovalov 1992). This acceleration takes place in the current layer, where the annihilation of azimuthal magnetic field occurs simultaneously.

Actual pulsars do not have parallel rotation and magnetic axes, and the problem of plasma ejection from such systems has not yet been solved. We shall thus use the $\gamma$-ray particle spectrum appropriate for the parallel case.

According to Bogovalov (1990b), the particles near the light cylinder are accelerated up to

$$
\gamma=\gamma_{0}+\frac{1}{2} e H X^{2} / \lambda m c \Omega
$$

where $\gamma$ is the Lorentz factor, $\lambda$ is the density ratio of $e^{+} e^{-}$plasma to the Goldreich density $n_{\mathrm{G}}=$ $H \Omega / 2 \pi e c$. It follows from the work of Gurevich and Istomin (1985) that $\lambda \simeq 10^{3}$, and the initial Lorentz factor of the particles, $\gamma_{0} \simeq 10^{4} . X$ is the distance expressed as a fraction of the light cylinder radius from the rotation axis to the base of the field line on the polar cap.

Eq.(2) as well as eq.(1) is obtained assuming that the density of electric current flowing across the polar cap is equal to the Goldreich current density $j_{a}=H \Omega / 2 \pi$ (Goldreich and Julian 1969). The second term in eq.(2) expresses the density of energy flux of the electromagnetic field on the field line emerging from the point on the polar cap which is at a distance $X$ from the rotation axis. This value is obtained on the condition that the total field energy flux is transformed into an energy flux of particles (Bogovalov 1990). 
The flux of particles ejected from the polar cap per unit time is (Ruderman and Sutherland 1975)

$$
d N / d t=2 \pi n c(c / \Omega)^{2} d X^{2} .
$$

Later we shall consider $\lambda$ and $H$ to be independent of $X$. Then from eqs.(2) and (3) we can obtain the spectrum of the particles

$$
d N / d t d \gamma=\left\{\begin{aligned}
0 ; & \gamma<\gamma_{0} \\
2 \lambda^{2} m c^{3} / e^{2} ; & \gamma_{0} \leq \gamma \leq \gamma_{\max } \\
0 ; & \gamma>\gamma_{\max }
\end{aligned}\right.
$$

In eqs.(1) and (4) the presence of the two poles of the pulsar is taken into account. The value $\gamma_{\max }$ is determined by eq. $(3)$, where $(R \Omega / c)^{3 / 2}$ should be substituted for $X$. The integral $\int_{0}^{\infty} \gamma m c^{2}(d N / d t d \gamma) d \gamma$ represents the energy flux from the pulsar [defined by eq.(1)] plus an additional contribution associated with the initial kinetic energy of the particles.

\section{$\gamma$-ray generation}

$\gamma$-ray radiation of extremely high energies from the Crab and Vela pulsars can be accounted for as a result of scattering of relativistic electrons accelerated near the light cylinder from thermal photons radiated by the neutron star. Observations show that the temperature of PSR 0833-45 is $T=$ $(0.6-1.0) \times 10^{6} \mathrm{~K}$ (Harnden et al. 1985, Ögelman and Zimmerman 1989). For PSR $0531+21$ only an upper limit $T<2.5 \times 10^{6} \mathrm{~K}$ has been obtained (Harnden and Seward 1984). Bearing in mind that the latter is younger than PSR 0833-45, its temperature must not be less than some $10^{6} \mathrm{~K}$.

The energy of $\gamma$-ray photons produced by inverse Compton scattering of ultra relativistic electrons (and positrons) on soft thermal photons depends on the parameter $\omega \gamma$, where $\omega$ is the energy of thermal photons in $m c^{2}$ units. Provided that $\omega \gamma \gg 1$, then every collision produces a quantum with $E_{\gamma}$ of the order of the energy of the incident electron (Ginzburg 1982). Keeping this in mind, we use eq.(4) to derive the integral spectrum of $\gamma$ quanta near the Earth as follows

$$
\begin{aligned}
N(>E)= & 0.16 \frac{r_{\mathrm{e}} \Omega R^{2} \lambda^{2} T^{2}}{\lambda^{3} R_{*}^{2} \theta}\left[\ln \left(6 T \gamma_{\max }\right)+0.5\right] \\
& \times \ln \left(\frac{\gamma_{\max } m c^{2}}{E_{\gamma}}\right)
\end{aligned}
$$

Here $R_{*}$ is the distance to the source, $r_{\mathrm{e}}$ the classical electron radius, $\lambda$ the Compton length, and $\theta$ the angular size of the radiation cone, which can be determined from the $\gamma$-ray light curve of the pulsar. Eq.(5) assumes that the density of thermal photons near the light cylinder $n_{\mathrm{ph}}$ is $0.244(T / \lambda)^{3}(R \Omega / c)^{2}$, where $T$ is the temperature in $m c^{2}$ units.

A comparison of the theoretical spectra [eq.(4)] with the observation data on the Crab and Vela pulsars is given in figure 1. For the Crab pulsar:

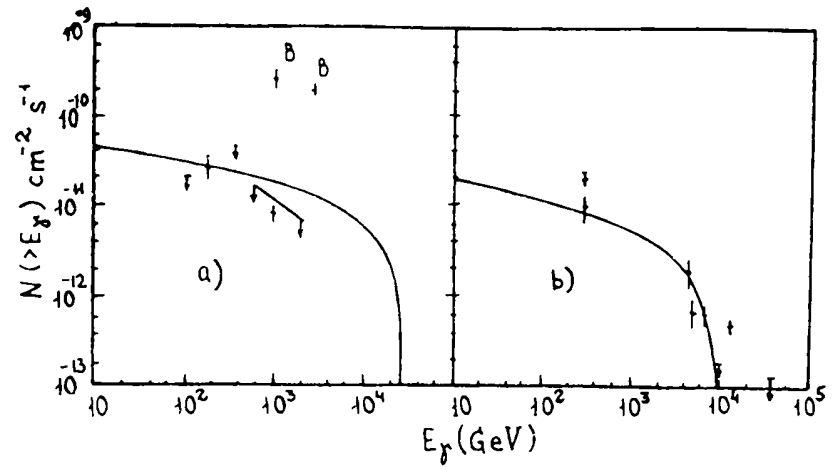

Figure 1 Theoretical spectra and observations of (a) the Crab and (b) Vela in high energy $\gamma$-rays. Crab burst-like events are marked by the letter " $B$ ".

$L=4 \times 10^{38} \mathrm{erg} / \mathrm{s}, R_{*}=2 \mathrm{kpc}, \theta=0.2, \lambda=10^{3}$, $T=10^{6} \mathrm{~K}, H=5.8 \times 10^{12} \mathrm{G}$. For the Vela pulsar: $L=10^{37} \mathrm{erg} / \mathrm{s}, R=0.5 \mathrm{kpc}, \theta=0.6, \lambda=500$, $T=10^{6} \mathrm{~K}, H=6.6 \times 10^{12} \mathrm{G}$. On the whole there is good agreement between the theory and observations, given inaccuracies in the experimental data, some uncertainty in the coefficient $\lambda$, and the circumstance that the angle between the magnetic and rotation axes is in reality far different from zero.

Observations of the Crab pulsar in $\gamma$ rays with $E_{\gamma} \simeq 1 \mathrm{TeV}$ reveal sudden "burst-type" increases superposed on a stationary, periodic background flux. The results of such observations are marked by a "B" in figure 1. Eq.(4) indicates that the intensity of $\gamma$-radiation strongly depends both on $T$ and on $\lambda$. A 4-5 times increase in either of these quantities could provide the observed growth of the flux. The coefficient $\lambda$ is defined by the structure and magnitude of the magnetic field at distances of the order $R$ from the pulsar (Gurevich and Istomin 1985b). Appreciable changes are unlikely. A possible reason for surface temperature increases may be the energy released from crustal stresses during a starquake (Manchester and Taylor 1977). Such phenomena apparently accompany timing "glitches", although no evidence correlating $\gamma$-ray bursts with glitches yet exists. In Vela observations, only one soft X-ray burst was detected (Smith and Pounds 1977) which was not accompanied by a glitch. This can mean that only soft X-ray bursts and bursts of super-high energy $\gamma$-rays are correlated. Correlations with glitches will then exist only if they are accompanied by X-ray bursts.

A connection between X-ray and $\gamma$-ray bursts may be observable for the Crab pulsar, because increases of the surface temperature of $(4-5) \times 10^{6} \mathrm{~K}$ exceed its measured upper limit of $2.5 \times 10^{6} \mathrm{~K}$. 